\title{
Changes in Exodeviation after the Monocular Occlusion Test in Adult Patients with Intermittent Exotropia and Its Association with the Level of Control
}

\author{
Jinho Shin, Won Jae Kim \\ Department of Ophthalmology, Yeungnam University Medical Center, Daegu, Korea
}

Purpose: We investigated the changes in ocular deviation after the monocular occlusion test in adults with intermittent exotropia and evaluated its association with the level of control.

Methods: We retrospectively enrolled adults (aged $\geq 18$ years) with intermittent exotropia who visited our clinic between September 2015 and May 2019. Patients with basic intermittent exotropia with a distant deviation within 10 prism diopters (PD) of the near deviation were included. The largest ocular deviations obtained before and after 1 hour of monocular occlusion were compared. The level of control was measured using the LACTOSE (Look and Cover, then Ten seconds of Observation Scale for Exotropia) control scoring system.

Results: Forty-six consecutive adult patients (28 males, 18 females; mean age, 34.3 years) were enrolled. The mean ocular deviation was 36.3 PD (range, 18 to 5 PD) at distant fixation and 38.5 PD (range, 18 to 80 PD) at near fixation, which increased significantly to $38.5 \mathrm{PD}(p=0.043)$ and $41.1 \mathrm{PD}(p=0.011)$, respectively, after monocular occlusion. The mean ocular deviation increased $\geq 5$ PD in 14 (30.4\%) and 15 (32.6\%) patients at distant and near fixation, respectively. The level of control was measured in 30 patients. A higher degree of near control was significantly associated with an increase of $\geq 5$ PD in near fixation after the test ( $p=0.009$ for a near control score $\leq 2$ ).

Conclusions: The monocular occlusion test may help to determine the largest ocular deviation in adults with intermittent exotropia. Approximately one-third of patients exhibited an increase in ocular deviation $\geq 5$ PD. Patients exhibiting good control were more likely to manifest an increase in the ocular deviation.

Key Words: Exotropia, Strabismus

The monocular occlusion test is a diagnostic method that measures ocular deviation in patients with strabismus [1-5]. Monocular occlusion is used to delineate intermittent exo-

Received: June 15, 2020 Final revision: October 7, 2020 Accepted: October 20, 2020

Corresponding Author: Won Jae Kim, MD. Department of Ophthalmology, Yeungnam University Medical Center, 170 Hyeonchung-ro, Nam-gu, Daegu 42415, Korea. Tel: 82-53-620-3444, Fax: 82-53-626-5936, E-mail: eyekwj@ynu.ac.kr tropia into the true divergence and pseudo-divergence excess types via by blocking the fusional stimuli in patients with intermittent exotropia [6]. Furthermore, it can be used to determine the largest ocular deviation in patients with intermittent exotropia [6]. Postoperative exodrift and recurrent intermittent exotropia can develop progressively following surgical treatment for intermittent exotropia [7]. The largest ocular deviation is the target of surgery in patients with intermittent exotropia $[6,8]$. Therefore, monocu- 
lar occlusion may aid in the planning of appropriate surgical treatment, thus ensuring a favorable surgical outcome.

Previous studies on the changes in ocular deviation after the monocular occlusion test usually included pediatric patients with intermittent exotropia [2-5]. Intermittent exotropia in adults may differ clinically from that in children [9]. The number of adult patients who underwent surgical treatment for intermittent exotropia has increased recently [10]. However, no study has evaluated the effect of the monocular occlusion test in only adult patients with intermittent exotropia. Moreover, we focused on the association between the level of control and change in exodeviation after the monocular occlusion test. The ocular deviation angle may vary depending on the level of control during follow-up $[6,8,11]$. Therefore, we evaluated the changes in ocular deviation after the monocular occlusion test in adult patients with intermittent exotropia and their association with the level of control.

\section{Materials and Methods}

The study protocol adhered to the tenets of the Declaration of Helsinki, and the need for informed consent was waived owing to the retrospective nature of the study by our institution's ethics board (2019-05-049). We retrospectively included all adult patients (aged $\geq 18$ years) with intermittent exotropia who visited our clinic between September 2015 and May 2019. Patients with a history of intraocular or strabismus surgery, those with nystagmus, amblyopia, or any neurological impairment were excluded. Patients with basic intermittent exotropia with a distance deviation within 10 prism diopters (PD) of the near deviation were included in this study.

\section{Preoperative evaluation}

All patients underwent complete ophthalmologic examination at their first visit, including visual acuity and ocular alignment assessment, slit lamp biomicroscopy, refraction testing, fundus examination, and stereoacuity testing. The best-corrected visual acuity was measured. The angle of deviation was measured using the prism cover test at 6-m and 33-cm fixation. Each patient was provided with an ocular patch at the first visit; all patients were informed that we planned to occlude the non-dominant eye for 1 hour at the next visit to measure the largest angle of deviation. The level of control was measured using the Look and Cover, then Ten seconds of Observation Scale for Exotropia (LACTOSE) control scoring system [12], which explores both distant and near control (5-point scales; range, 0 to 4 for each), yielding total scores of 0 to 8 . Higher scores were indicative of poor control. Controllability was defined as the subjective awareness of exotropia and instinctive ability to correct ocular deviation. All clinical examinations were performed by one clinician (WJK). The stereoacuity test was performed using the Lang I (Lang-Stereotext AG, Küsnacht, Switzerland) and Stereo Fly (Stereo Optical Co., Chicago, IL, USA) stereotests.

\section{Statistical analysis}

Continuous data were expressed as means \pm standard deviations and categorical data were expressed as counts and percentages. The paired $t$-test was used to evaluate the differences within each group. The largest ocular deviations before and after monocular occlusion test were compared. The unpaired $t$-test and chi-squared test were used to compare the clinical parameters of patients who exhibited an increase of $\geq 5$ PD after occlusion, and others who exhibited no significant increase or a decrease in the ocular deviation. The logistic regression test was employed to evaluate the association between clinical factors and an increase $\geq 5$ PD after monocular occlusion test. Data were analyzed using IBM SPSS Statistics ver. 20.0 (IBM Corp., Armonk, NY, USA). A $p$-value $<0.05$ was considered statistically significant.

\section{Results}

Basic characteristics of adults with intermittent exotropia

Forty-six consecutive patients (28 males, 18 females; mean age, 34.3 years) were enrolled in this study. Their basic characteristics are presented in Table 1. The ocular deviation was $36.3 \pm 14.5 \mathrm{PD}$ (range, 18 to $75 \mathrm{PD}$ ) at distant fixation and $38.5 \pm 15.3 \mathrm{PD}$ (range, 18 to $80 \mathrm{PD}$ ) at near fixation. The level of control was measured for 30 patients. The mean distant, near, and combined control scores were 3.3, 3.1, and 6.4, respectively. The control scores exhibited 
Table 1. Basic characteristics of the adult patients with intermittent exotropia $(n=46)$

\begin{tabular}{lc}
\hline Characteristics & Value \\
\hline Mean age of patients (yr) & $34.3 \pm 16.5(18$ to 78$)$ \\
Sex (male : female) & $28: 18$ \\
Mean age of onset of exotropia $(36 / 46, y r)$ & $21.9 \pm 15.9(1$ to 63$)$ \\
Amount of ocular deviation (prism diopters) & $36.3 \pm 14.5(18$ to 75$)$ \\
$\quad$ Distant & $38.5 \pm 15.3(18$ to 80$)$ \\
Near & $3.3 \pm 1.1(1$ to 4$)$ \\
Level of control, control score $(30 / 46)$ & $3.1 \pm 1.2(1$ to 4$)$ \\
$\quad$ Distant & $6.4 \pm 2.2(2$ to 8$)$ \\
Near & $9 / 46(19.6)$ \\
Overall & \\
Patients with controllability & $-2.14 \pm 2.95(-12.88$ to 1.13$)$ \\
Spherical equivalent refractive errors & $-2.01 \pm 2.65(-11.38$ to 1.50$)$ \\
$\quad$ Right eye & \\
Left eye & $28 / 44(63.6)$ \\
Results of the stereotest & $32 / 44(72.7)$ \\
Lang I test, passed & \\
Stereo Fly Stereotest $(\leq 400$ arcsec) & \\
\hline
\end{tabular}

Values are presented as mean \pm standard deviation (range), number, or number (\%); The level of control was measured using the LACTOSE (Look and Cover, then Ten seconds of Observation Scale for Exotropia) control scoring system, which explores both distant and near control (5-point scales; range, 0 to 4 for each) and yields total scores of 0 to 8 . A higher score indicated poorer control. Controllability was defined as the subjective awareness of exotropia and instinctive ability to correct the ocular deviation.

a significant correlation with the results of the Lang I test ( $p=0.017$ for distant control, $p=0.004$ for near control; linear-by-linear association). The number of patients who "failed" the Lang I test increased in proportion to the increase in the distant and near control scores (reflecting poorer control). The near control scores were correlated with the Stereo Fly stereotest scores ( $p=0.022$ at $\leq 400$ arc seconds; linear-by-linear association). Although the distant control scores were also correlated with the Stereo Fly stereotest scores, statistical significance was not attained ( $p=$ $0.063)$. Nine patients $(19.6 \%, 9 / 46)$ exhibited controllability of exotropia.

\section{Changes in ocular deviation after the monocular occlu- sion test in adults with intermittent exotropia}

The ocular deviation at distant fixation increased significantly from $36.3 \pm 14.5$ to $38.5 \pm 14.7 \mathrm{PD}$ after monocular occlusion ( $p=0.043$, paired $t$-test) (Fig. 1). The ocular deviation at near fixation increased significantly from $38.5 \pm$

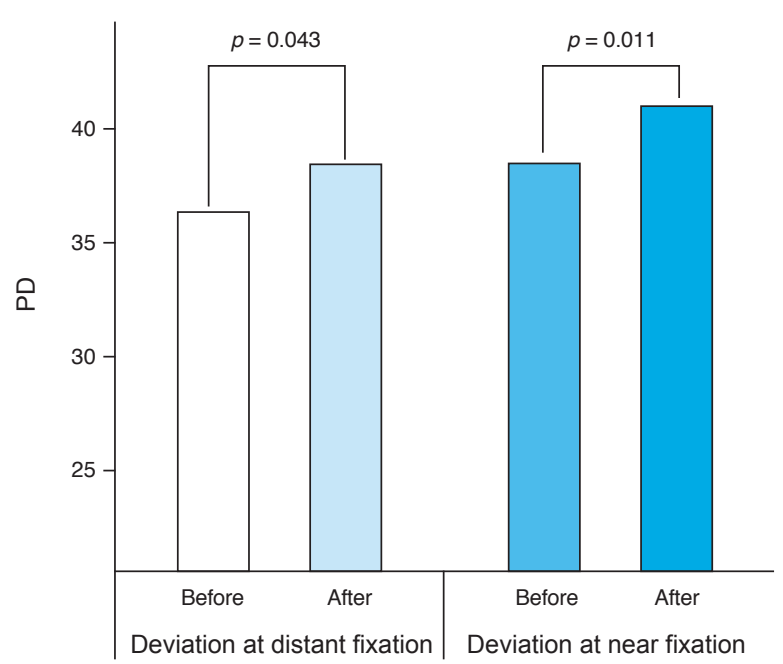

Fig. 1. Changes in ocular deviation at distant and near fixation after the monocular occlusion test. The ocular deviation at both distant and near fixation increased significantly after the monocular occlusion test. The ocular deviation at distant fixation increased significantly from $36.3 \pm 14.5$ to $38.5 \pm 14.7$ prism diopters (PD) after monocular occlusion ( $p=0.043$, paired $t$-test). The ocular deviation at near fixation increased significantly from $38.5 \pm 15.3$ to $41.1 \pm 13.8 \mathrm{PD}$ after the monocular occlusion test $(p=0.011)$. 
15.3 to $41.1 \pm 13.8 \mathrm{PD}$ after monocular occlusion $(p=0.011)$ (Fig. 1). The mean change in ocular deviation after occlusion was $2.2 \pm 7.2 \mathrm{PD}$ (range, -5 to $35 \mathrm{PD}$ ) at distant fixation and $2.5 \pm 6.5 \mathrm{PD}$ (range, -5 to $30 \mathrm{PD}$ ) at near fixation. The largest difference between the ocular deviations before and after monocular occlusion was 35 PD for distant fixation and 30 PD for near fixation. Fig. 2 shows the percentages of patients with reduction, no significant change, or increase $\geq 5$ PD in ocular deviation after the monocular occlusion test. Fourteen patients $(30.4 \%, 14 / 46)$ exhibited increases $\geq 5$ PD at distant fixation, and 15 patients $(32.6 \%$, $15 / 46$ ) exhibited increases $\geq 5 \mathrm{PD}$ at near fixation.

\section{Associations between the changes in ocular deviation after the monocular occlusion test and level of control}

Fig. 3 depicts the percentages of patients with an increase in ocular deviation $\geq 5$ PD after the monocular occlusion test based on the level of control. The percentage of such increases at near fixation rose significantly with the fall in the near control scores (reflecting better control) ( $p=0.009$ for near control, chi-square test). Although the percentage of patients with increases $\geq 5 \mathrm{PD}$ at distant fixa-

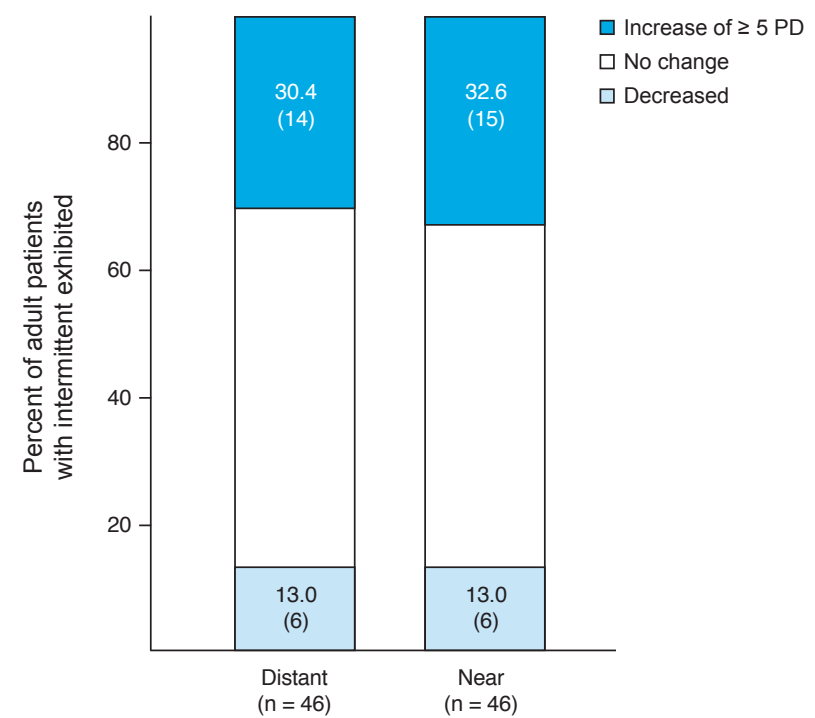

Fig. 2. Percentages of patients who showed reduction, no significant change, or increase of $\geq 5$ prism diopters (PD) in ocular deviation after the monocular occlusion test. Fourteen patients (30.4\%, $14 / 46)$ exhibited an increase of $\geq 5$ PD in ocular deviation at distant fixation, and 15 patients $(32.6 \%, 15 / 46)$ exhibited an increase of $\geq 5 \mathrm{PD}$ in ocular deviation at near fixation after the monocular occlusion test. tion increased with a fall in the distant control scores, it failed to achieve statistical significance ( $p=0.225$ for distant control). The mean ocular deviations increased by $\geq 5$ $\mathrm{PD}$ in 7 of 9 patients who exhibited controllability $(77.8 \%$, 7 / 9). Better near control was significantly associated with an increase of $\geq 5$ PD at near fixation (odds ratio, 0.10; 95\% confidence interval, 0.018 to $0.561 ; p=0.009$ for a near control score $\leq 2$; logistic regression test). No other clinical factor (mean age, sex distribution, mean age at the onset of exotropia, or spherical equivalent refractive error) differed between patients exhibiting an increase $\geq 5$ PD and those with no significant changes or a decrease in the ocular deviation (all $p>0.05$ ). No clinical factor was associated with an increase in ocular deviation $\geq 5$ PD after the monocular occlusion test (all $p>0.05$, logistic regression test).

\section{Discussion}

Ocular deviation increased significantly after the monocular occlusion test in adults with intermittent exotropia.

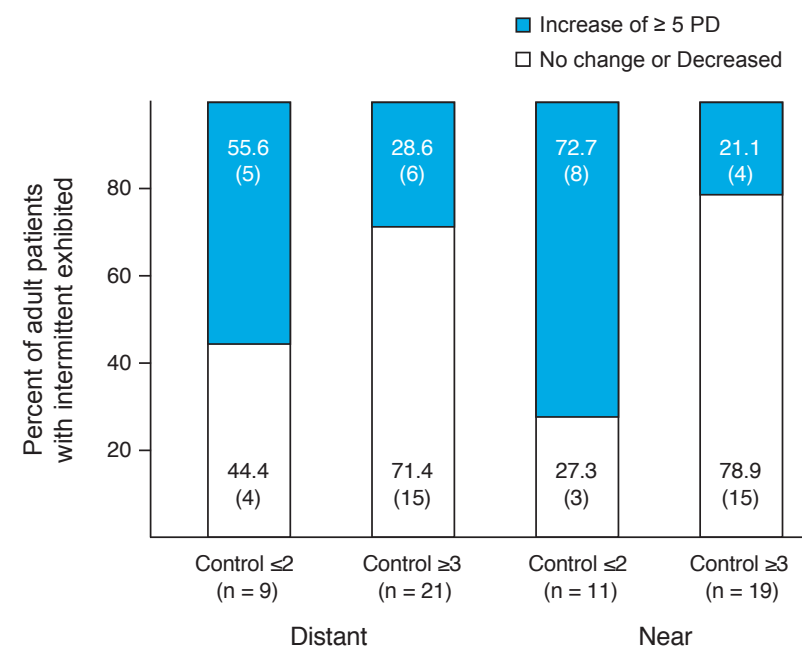

Fig. 3. Percentage of patients with an increase of $\geq 5$ prism diopters (PD) after the monocular occlusion test according to the level of control. The level of control was measured using the LACTOSE (Look and Cover, then Ten seconds of Observation Scale for Exotropia) control scoring system, which explores both distant and near control (5-point scales; range, 0 to 4 for each) and yields total scores of 0 to 8 . A higher score was indicative of poorer control. The level of control was assessed in 30 of 46 adult patients with intermittent exotropia. The percentage of patients with an increase of $\geq 5$ PD after the monocular occlusion test increased with the decrease in the control scores showing a better level of control ( $p=0.225$ for distant control, $p=0.009$ for near control). 
Approximately one-third of patients exhibited increases $\geq 5$ PD and such elevations were more likely to occur in patients who exhibited good control. The monocular occlusion test should be performed for adults with intermittent exotropia, especially those exhibiting better control, to determine the largest ocular deviations.

Patients with intermittent exotropia evidence varying degrees of ocular deviation during follow-up. Kim and Kim [11] found that over half of such patients exhibited variable deviation angles preoperatively. The largest angle of deviation should be assessed to avoid undercorrection and ensure a favorable surgical outcome. The monocular occlusion test determines the largest ocular deviation by dissipating any fusional convergence that may affect deviation and is often recommended for clinical use $[6,8]$.

Previous studies found that ocular deviation increased significantly after the monocular occlusion test $[1,2]$. Lin et al. [3] showed that a 1-hour long diagnostic occlusion test elicited the largest near deviation angle in patients with intermittent exotropia. However, most previous studies included pediatric patients with intermittent or other types of exotropia. The frequency of strabismus surgery in adult patients has increased recently, owing to its functional and psychosocial benefits [10]. No study has evaluated the effect of the monocular occlusion test only in adults with intermittent exotropia. This study included adult patients with basic exotropia, which is the most common type of intermittent exotropia [6]. We also evaluated the association between the level of control and change in ocular deviation after the monocular occlusion test.

Adults with intermittent exotropia exhibited increased ocular deviation after the monocular occlusion test (Fig. 1), which is consistent with the results of previous studies. The mean increase in ocular deviation was 2.2 PD at distant fixation and 2.5 PD at near fixation, which is apparently minimal. However, approximately one-third of patients exhibited increases $\geq 5$ PD. The largest increase was 30 PD. The surgical outcome may be unfavorable if such changes are not determined preoperatively. Six patients $(13.0 \%, 6 / 46)$ exhibited decreased ocular deviation after distant or near fixation. Previous studies also found that a few patients exhibited such decreases after the monocular occlusion test [4,5], which is perhaps explained by the variation in the level of control [11]. Even 1 hour of monocular occlusion may not dissipate fusion if the level of control is good, thus decreasing ocular deviation. Han et al. [4] opined that the monocular occlusion test had a limited role in determining the maximum angle of deviation compared to several other examinations.

Adult patients with relatively better control may be more likely to exhibit an increase in ocular deviation after the monocular occlusion test. More than half the patients with controllability, who were considered to have a relatively better level of control exhibited an increase in ocular deviation $\geq 5$ PD after the monocular occlusion test. A better level of control was associated with better fusional capacity and greater variability in the ocular deviation in patients with intermittent exotropia [11,13]. Patients with relatively better fusional potential are more likely to maintain normal ocular alignment for some time, which can easily mask the full extent of ocular deviation, thus creating variability. A previous study showed that the surgical outcomes of intermittent exotropia were better in patients whose preoperative measurements were variable rather than consistent [11], suggesting that patients with better fusional convergence exhibited greater variability in ocular deviations, which leads to better surgical outcomes. Therefore, the monocular occlusion test should be performed in adults with intermittent exotropia, especially those exhibiting better control, to determine the largest ocular deviations.

Arnoldi and Reynolds [14] showed that patients with poor control over distant fixation were unlikely to show tenacious distance fusion on the patch test, and that this test may be avoided in such patients. Adult patients with intermittent exotropia may exhibit a lower degree of fusion than pediatric patients [9]. In our study, the mean control score was higher, which represented a poorer level of control, compared to previous studies that included pediatric patients with intermittent exotropia [12]. Five of 20 patients (25\%) with distance control scores of 4 exhibited an increase in distant fixation $\geq 5$ PD after the monocular occlusion test. The test is non-invasive and relatively short (1 hour) [2]. We recommend that the monocular occlusion test should be performed even in adult patients exhibiting poor control.

Our study has certain limitations. First, the surgical outcomes based on the changes in ocular deviation after the monocular occlusion test could not be evaluated because not all patients underwent surgery, and the postoperative follow-up period was too short for the surgical patients. We endeavor to analyze the surgical outcomes in a future 
study. Second, the variability in the level of control can affect the change in ocular deviation, because the measurements before and after the monocular occlusion test were not performed on the same day. The level of control may vary during the day and may even change within minutes [15]. Moreover, we analyzed the level of control measured at one time point, i.e., at the first visit. Future studies should include serial measurements. Third, not all patients were evaluated using the LACTOSE scoring system, which became available only in 2017 [12], since we included patients treated before this time.

\section{Conflict of Interest}

No potential conflict of interest relevant to this article was reported.

\section{References}

1. Kushner BJ. The distance angle to target in surgery for intermittent exotropia. Arch Ophthalmol 1998;116:189-94.

2. Gurlu VP, Erda N. Diagnostic occlusion test in intermittent exotropia. J AAPOS 2008;12:504-6.

3. Lin S, Li FJ, Wang LH. Comparative measurements of exodeviations in intermittent exotropia. Zhonghua Yan Ke Za Zhi 2013;49:609-14.

4. Han JM, Yang HK, Hwang JM. Efficacy of diagnostic monocular occlusion in revealing the maximum angle of exodeviation. Br J Ophthalmol 2014;98:1570-4.

5. Jung EH, Kim SJ, Yu YS. Comparison of the characteris- tics of patients with intermittent exotropia according to response to diagnostic monocular occlusion. Jpn J Ophthalmol 2018;62:243-8.

6. Kushner BJ. Strabismus. 1st ed. Gewerbestrasse: Springer; 2017. p. 73-95.

7. Kim WJ, Kim MM. The clinical course of recurrent intermittent exotropia following one or two surgeries over 24 months postoperatively. Eye (Lond) 2014;28:819-24.

8. Wright KW, Mocan MC. Exotropia. In: Wright KW, Strube YN, editors. Pediatric ophthalmology and strabismus. 3rd ed. New York: Oxford University Press; 2012. p. 306-16.

9. Jung JW, Lee SY. A comparison of the clinical characteristics of intermittent exotropia in children and adults. Korean J Ophthalmol 2010;24:96-100.

10. Szigiato AA, Caldwell M, Buys YM, et al. Population trends in adult strabismus surgery. Can J Ophthalmol 2019;54:501-8.

11. Kim WJ, Kim MM. Variability of preoperative measurements in intermittent exotropia and its effect on surgical outcome. J AAPOS 2017;21:210-4.

12. Kim H, Kim DH, Ahn H, Lim HT. Proposing a new scoring system in intermittent exotropia: towards a better assessment of control. Can J Ophthalmol 2017;52:235-9.

13. Hatt SR, Leske DA, Holmes JM. Awareness of exodeviation in children with intermittent exotropia. Strabismus 2009;17:101-6.

14. Arnoldi KA, Reynolds JD. Assessment of amplitude and control of the distance deviation in intermittent exotropia. J Pediatr Ophthalmol Strabismus 2008;45:150-3.

15. Hatt SR, Mohney BG, Leske DA, Holmes JM. Variability of control in intermittent exotropia. Ophthalmology 2008;115:371-6. 